\title{
Determinación de parámetros morfológicos por resonancia magnética de tumores rectales para establecer un sistema de puntuación de rectometría (R.E.C.T.A.L. score) que nos permita estimar el grado de complejidad quirúrgica
}

\author{
Determination of morphological parameters by magnetic resonance of \\ rectal tumors to establish a rectometry scoring system (R.E.C.T.A.L. \\ score) that allows us to estimate the degree of surgical complexity
}

Verónica Argüello Ramírez, ${ }^{*}$ María de Guadalupe Gómez Pérez ${ }^{\ddagger}$

\begin{abstract}
Citar como: Argüello RV, Gómez PMG. Determinación de parámetros morfológicos por resonancia magnética de tumores rectales para establecer un sistema de puntuación de rectometría (R.E.C.T.A.L. score) que nos permita estimar el grado de complejidad quirúrgica. Acta Med. 2021; 19 (1): 21-24. https://dx.doi.org/10.35366/98565
\end{abstract}

\section{Resumen}

Objetivo: Describir las características morfológicas por resonancia magnética de tumores rectales para establecer un sistema de puntuación de rectometría (R.E.C.T.A.L score) que permita estimar el grado de complejidad quirúrgica. Material y métodos: Se realizó un estudio observacional, descriptivo, transversal ambispectivo seleccionando una muestra de pacientes que acudieron al Hospital Ángeles Pedregal del mes de marzo de 2017 al mes de diciembre de 2019. Incluyendo a todos aquellos pacientes con diagnóstico de carcinoma rectal a los que se les hubiera practicado resonancia magnética se les aplicó el instrumento denominado R.E.C.T.A.L score (R.E.C.T.A.L RM HAP/2019) para medir variables cualitativas y cuantitativas y así determinar el riesgo quirúrgico. Resultados: De los 48 pacientes que se ingresaron al estudio, 16 (33.3\%) de ellos tuvieron una complejidad quirúrgica baja, 16 (33.3\%) moderada y 16 (33.3\%) alta. Conclusiones: La puntuación propuesta para la complejidad quirúrgica no se ha realizado antes para este tipo de tumores; sin embargo, para tumores renales se ha aceptado su empleo. En nuestra población, en la que tenemos pacientes que presentaron tumores en

\section{Abstract}

Objective: To describe the morphological characteristics of rectal tumors by magnetic resonance imaging (MRI) in order to establish a rectometry scoring system (R.E.C.T.A.L. score) that enables the surgical complexity grading estimation. Material and methods: An observational, descriptive, cross-sectional, ambispective study was carried out selecting a sample of patients that came to the Hospital Ángeles Pedregal from March 2017 to December 2019. Including all patients diagnosed with rectal carcinoma who would have undergone MRI, the instrument referred to as R.E.C.T.A.L. score (R.E.C.T.A.L. RM $\mathrm{HAP} / 2019)$ was applied to patients to measure qualitative and quantitative variables, thus determine surgical risk. Results: Of the 48 patients that entered the study, $16(33 \%)$ of them had a low surgical complexity, 16 (33\%) moderate and 16 (33\%) high. Conclusions: The proposed punctuation for surgical complexity has not been accepted. In our population that includes patients with rectal tumors assessed by MRI, specific modifications were made for this type of injury and we found that provides specific details that can be used by surgeons to perform an adequate surgical approach and to complement it with adjunctive therapy.

\footnotetext{
* Médico Residente del Curso de Alta Especialidad en Resonancia Magnética cuerpo completo, Facultad de Medicina de la UNAM.

‡ Jefe del Servicio de Resonancia Magnética.
}

Hospital Ángeles Pedregal. Ciudad de México, México.
Correspondencia:

Dra. Verónica Argüello Ramírez

Correo electrónico: ramirez961202@yahoo.com.mx

www.medigraphic.com/actamedica

Aceptado: 30-03-2020. 
recto valorados por resonancia, se realizaron modificaciones específicas para este tipo de lesión y encontramos que ofrece detalles específicos que pueden ser utilizados por el cirujano para realizar un adecuado abordaje quirúrgico y decidir complementarlo con terapia adyuvante.

Palabras clave: Cáncer de recto, R.E.C.T.A.L. score, resonancia magnética.

\section{INTRODUCCIÓN}

El cáncer de recto es una patología con elevada incidencia en nuestro medio y constituye la tercera neoplasia tanto en varones como en mujeres en México tras el cáncer de próstata y pulmón, así como el de mama y cervicouterino, respectivamente. ${ }^{1}$ Aproximadamente una de cada tres personas muere por esta causa. Los factores de riesgo tanto genéticos como ambientales están fuertemente asociados con el desarrollo de esta patología.

Los síntomas son comunes e importantes en cáncer tardío cuando el pronóstico es pobre y son menos comunes y menos obvios en estadio temprano de la enfermedad. Los síntomas comunes incluyen dolor abdominal, sangrado rectal, alteración de los hábitos intestinales y pérdida involuntaria de peso. En el cáncer avanzado, particularmente con metástasis, puede haber caquexia caracterizada por una tétrada sintomática de pérdida involuntaria de peso, anorexia, debilidad muscular y sentimiento de pérdida de salud. ${ }^{2}$

La Sociedad Americana de Cáncer recomienda diferentes métodos de escrutinio que van desde procedimientos no invasivos, como sangre oculta en heces, hasta procedimientos semiinvasivos, como la colonoscopia. ${ }^{3}$ El desafío actual de la cirugía de cáncer de recto es realizar la misma con fines curativos, con la mínima morbilidad y con los índices más bajos de recurrencia. Entre los pacientes que han sido sometidos a resección por enfermedad localizada la supervivencia a los cinco años es de 90\% y de sólo 65\% si están presentes nódulos linfáticos metastásicos.

Estos pacientes presentan un alto riesgo de recurrencias locales pélvicas tras la resección quirúrgica, de 30 a $40 \%$ recurren dentro de los primeros dos a tres años. El éxito del tratamiento va a depender en gran medida de la obtención de un margen libre de tumor, el llamado margen de resección circunferencial. ${ }^{4}$ La resonancia magnética (RM) es la técnica de elección para estudiar la relación del tumor con la fascia mesorrectal y determinar el margen de resección circunferencial para poder evaluar la resecabilidad del tumor. ${ }^{5}$ Por lo que es importante determinar parámetros morfológicos por resonancia magnética de tumores rectales y establecer un sistema de puntuación de rectometría (R.E.C.T.A.L.
Keywords: Rectal cancer, R.E.C.T.A.L. score, magnetic resonance imaging. score) que nos permita estimar el grado de complejidad quirúrgica, para así determinar qué pacientes pueden ser tratados sólo con cirugía y cuáles requieren de un tratamiento neoadyuvante previo para promover la regresión del tumor. ${ }^{6}$

\section{Objetivos}

- Describir las características morfológicas por resonancia magnética de tumores rectales para establecer un sistema de puntuación de rectometría (R.E.C.T.A.L. score).

- Simplificar y estandarizar la terminología y el contenido de los informes de resonancia magnética mediante la puntuación de rectometría (R.E.C.T.A.L. score).

- Desarrollar un sistema de puntuación que permita estimar el grado de complejidad quirúrgica.

\section{MATERIAL Y MÉTODOS}

Se realizó un estudio observacional, descriptivo, transversal ambispectivo seleccionando una muestra de pacientes que acudieron al Hospital Ángeles Pedregal del mes de marzo de 2017 al mes de febrero de 2019 para la muestra del tipo retrospectivo y de pacientes para la muestra del tipo prospectivo que abarcó de marzo a diciembre de 2019. Incluyendo a todos aquellos pacientes con diagnóstico de carcinoma rectal a los que se les hubiera practicado resonancia magnética se les aplicó el instrumento denominado R.E.C.T.A.L score (R.E.C.T.A.L. RM HAP/2019) para medir variables cualitativas y cuantitativas y así determinar el riesgo quirúrgico de tumores rectales mediante esta técnica en nuestra población.

\section{RESULTADOS \\ Datos demográficos generales}

Fueron capturados los datos de 48 pacientes con diagnóstico de tumor rectal visualizado por resonancia magnética, con el protocolo establecido de los cuales 25\% (12 pacientes) corresponden al sexo femenino y $75 \%$ (36) al masculino. 
Tabla 1: Sistema de puntuación R.E.C.T.A.L. score.

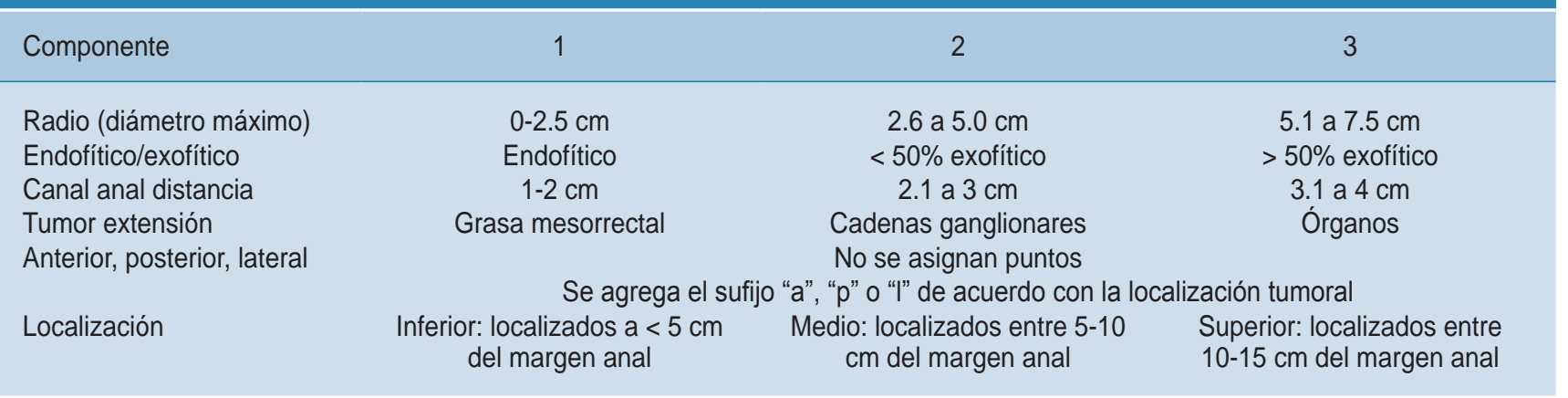

\section{Características generales}

El R.E.C.T.A.L. score se compone de seis características importantes desde el punto de vista quirúrgico, pero sólo cinco suman puntos para el cálculo de la rectometría (R.E.C.T.A.L.) en una escala de 1,2 y 3 puntos.

El sexto ítem, el $A$, agrega un sufijo (esto es, a de anterior, $p$ de posterior y / de bilateral) a la puntuación de acuerdo con la localización de la lesión. Si el tumor no encuadra en esas categorías, se agrega como sufijo una x (Tabla 1).

\section{Características particulares de los componentes}

Radio (R). Este componente representa el diámetro máximo del tumor en plano axial.

Relación endofítico/exofítico (E). Otra variable importante para la resecabilidad tumoral es la proporción del componente por fuera (exofítico) o por dentro (endofítico) de la lesión respecto del borde de ésta. Los tumores endofíticos se pueden resecar de manera más sencilla que aquellos que presentan un componente exofítico.

Canal anal (C). El componente $C$ denota la infiltración del tumor al canal anal. Esto es relevante para evaluar la resecabilidad del tumor y la necesidad de realizar reconstrucciones complejas.

Tumor extensión ( $\mathrm{T}$ ). El componente $T$ denota la extensión del tumor, ya sea limitado a la grasa mesorrectal, cadenas ganglionares o a órganos.

Localización anatómica (A). El componente $A$ no suma en el cálculo de la complejidad quirúrgica de la lesión; sin embargo, sirve para planear el abordaje quirúrgico, en esencia otorga información con más detalle al cirujano de la ubicación espacial de la lesión.

Localización (L). El componente $L$ se define por las líneas virtuales que dividen al recto en:

- Recto inferior: $<5 \mathrm{~cm}$ del margen anal.

- Recto medio: entre 5-10 cm.

- Recto superior: localizados entre 10-15 cm (Figura 1).

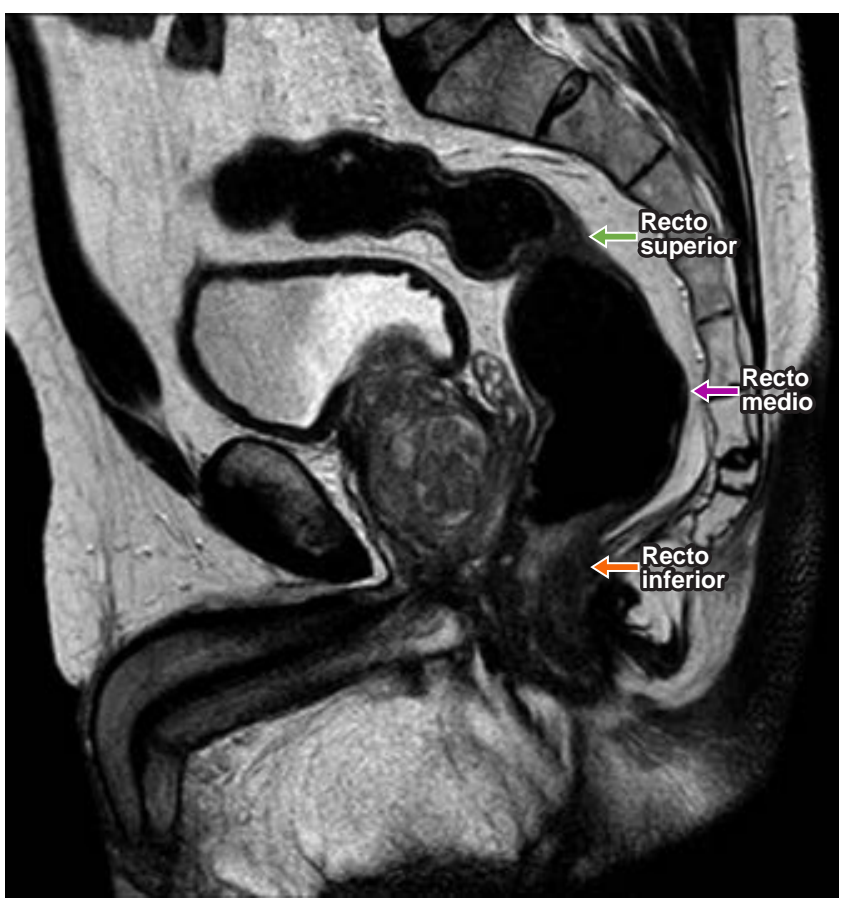

Figura 1: Resonancia magnética potenciada en T2 en plano sagital en la que se observa la localización de los tumores de recto.

Complejidad quirúrgica tumoral. Ésta la vamos a dividir en baja cuando el puntaje de la escala sea de cuatro a seis puntos, moderada de siete a nueve puntos y alta de 10 a 12 puntos.

\section{DISCUSIÓN}

El presente estudio tuvo la finalidad de implementar esta escala que ya se ha utilizado para evaluar la complejidad quirúrgica en tumores de origen renal; se adaptó para nuestros pacientes con diagnóstico de tumor rectal, la cual 
ayuda, en primera instancia, a presentar de manera ordenada y con más detalle los reportes radiológicos (en este caso de resonancia magnética) y, en segunda, a categorizar de una manera rápida para el cirujano cuáles son aquellos pacientes que presentarán mayores dificultades técnicas al momento de la cirugía, por ejemplo, el aumento en el puntaje en el RENAL score se ha asociado con un mayor tiempo quirúrgico, un incremento de tiempo de la isquemia y una mayor pérdida de sangre y por su parte los tumores de baja complejidad quirúrgica han presentado menos tiempo de isquemia; sin embargo, en nuestros pacientes no tenemos información sobre datos quirúrgicos, los cuales estarían reforzando la relación entre el R.E.C.T.A.L. score y la complejidad del tumor.

En nuestra población encontramos una distribución homogénea en cuanto a complejidad quirúrgica se refiere, observamos que aquellos pacientes con mayor puntaje presentaron un índice mayor de complicaciones en el caso específico de las fístulas, que es la complicación que se presenta de manera más frecuente en estos pacientes.

Asimismo, el uso de la escala se pretende implementar como orientación terapéutica, ya que a todos los pacientes se les otorga tratamiento quirúrgico; sin embargo, aquellos que se encuentran con una complejidad quirúrgica media y alta se les tendría, por las características del tumor, que asociar una terapia adyuvante, como son la radioterapia y quimioterapia, y de esta manera obtener la mejor respuesta posterior al tratamiento. ${ }^{7}$

\section{CONCLUSIONES}

La puntuación propuesta para la complejidad quirúrgica no se ha realizado antes para este tipo de tumores; sin embargo, para tumores renales se ha aceptado su empleo. En nuestra población, en la que tenemos pacientes que presentaron tumores en recto valorados por resonancia, se realizaron modificaciones específicas para este tipo de lesión y encontramos que ofrece detalles específicos que pueden ser utilizados por el cirujano para realizar un adecuado abordaje quirúrgico y decidir complementarlo con terapia adyuvante que provea al paciente de una mejor respuesta al tratamiento y por lo tanto una menor presentación de complicaciones, lo cual es común en estos pacientes.
La facilidad del puntaje en esta escala puede ofrecer una herramienta óptima para que los pacientes con este tipo de tumor puedan ser valorados por el médico radiólogo, dando la información necesaria al cirujano, unificando términos y una manera rápida de decisión en cuanto a la terapia complementaria, ya que en estos pacientes un mayor puntaje se correlaciona de manera positiva para lesiones más agresivas y por lo tanto se debe pensar en el empleo de radioterapia o quimioterapia previo al evento quirúrgico..$^{8,9}$

Como perspectiva del proyecto se pretende realizarlo en una población mayor a la obtenida en este estudio, asociado con datos quirúrgicos específicos para la correcta aplicación de la escala y de esta manera poder validarla.

\section{REFERENCIAS}

1. Ferlay J, Soerjomataram I, Dikshit R, Eser S, Mathers C, Rebelo M et al. Cancer incidence and mortality worldwide: sources, methods and major patterns in GLOBOCAN 2012. Int J Cancer. 2015; 136 (5): E359-E386.

2. American Cancer Society. Cancer facts and figures 2018. [Accessed May 1, 2018] Available in: https://www.cancer.org/ research/cancer-facts-statistics/all-cancer-facts-figures/cancerfacts-figures-2018.html

3. Bailey CE, Hu CY, You YN, Bednarski BK, Rodriguez-Bigas MA, Skibber JM et al. Increasing disparities in the age-related incidences of colon and rectal cancers in the United States, 1975-2010. JAMA Surg. 2015; 150 (1): 17-22.

4. Nagtegaal I, Gaspar C, Marijnen C, Van De Velde C, Fodde R, Van Krieken H. Morphological changes in tumour type after radiotherapy are accompanied by changes in gene expression profile but not in clinical behaviour. J Pathol. 2004; 204 (2): 183192.

5. Krook JE, Moertel CG, Gunderson LL, Wieand HS, Collins RT, Beart RW et al. Effective surgical adjuvant therapy for high-risk rectal carcinoma. N Engl J Med. 1991; 324 (11): 709-715.

6. Gastrointestinal Tumor Study Group. Prolongation of the disease-free interval in surgically treated rectal carcinoma. N Engl J Med. 1985; 312 (23): 1465-1472.

7. NIH consensus conference. Adjuvant therapy for patients with colon and rectal cancer. JAMA. 1990; 264 (11): 1444-1450.

8. Sauer R, Becker H, Hohenberger W, Rödel C, Wittekind C, Fietkau $\mathrm{R}$ et al. Preoperative versus postoperative chemoradiotherapy for rectal cancer. N Engl J Med. 2004; 351 (17): 1731-1740.

9. National Comprehensive Cancer Network. NCCN guidelines: version 2.2017-rectal cancer. September 10, 2017. [Accessed May 1, 2018] Available in: https://www.nccn.org/professionals/physician_gls/pdf/ rectal.pdf. 